\title{
Astronomical Archives of the Future: a Virtual Observatory
}

\author{
A.S. Szalay \\ Department of Physics and Astronomy, The Johns Hopkins University, \\ Baltimore, MD, 21218 \\ R.J. Brunner \\ Department of Astronomy, California Institute of Technology, \\ Pasadena, CA, 91125
}

\begin{abstract}
.
Astronomy is entering a new era as multiple, large area, digital sky surveys are in production. The resulting datasets are truly remarkable in their own right; however, a revolutionary step arises in the aggregation of complimentary multi-wavelength surveys (i.e. the cross-identification of a billion sources). Federating these different datasets, however, is an extremely challenging task. With this task in mind, we have identified several areas where community standardization can provide enormous benefits in order to develop the techniques and technologies necessary to solve the problems inherent in federating these large databases, as well as the mining of the resultant aggregate data. Several of these areas are domain specific, however, the majority of them are not. We feel that the inclusion of non-astronomical partnerships can provide tremendous insights.
\end{abstract}

\section{Introduction}

There is a data avalanche coming in astronomy, several large projects are under way which will each produce many Terabytes of catalogued data, covering a large part of the sky. Many of these catalogs will cover different wavebands, from the X-rays to optical/infrared all the way to the radio. We believe, that astronomy is about to undergo a major paradigm shift, with data sets becoming larger and larger, and more homogeneous, for the first time designed in the top-down fashion. In a few years it will be much easier to 'dial-up' a part of the sky, when an astronomer needs a rapid observation than wait for several month or a year to access a (sometimes quite small) telescope. With the advent of inexpensive storage technologies and the availability of high speed networks (vBNS, Abilene, Internet 2), the concept of multiple, Terabyte size databases interoperating in a seamless fashion is not an outlandish idea any more. More and more catalogs will be added and linked to the existing ones, query engines will be more sophisticated, and astronomers will have to be just as familiar with mining data as with observing on telescopes. Soon it may become more cost 
effective to spend resources on integrating all the data archives into a National Virtual Observatory, than building another of the traditional observatories.

It is important to understand, that today's approaches of accessing astronomical data do not scale well into the Terabyte regime - brute force does not work! Let us assume a hypothetical 500 Gigabyte data set. The most popular data access technique today is the World Wide Web. Most university sites can receive data at the typical bandwidth of about $15 \mathrm{kbytes} / \mathrm{sec}$. The transfer time for this data set would be 1 year! If the data is residing locally within the building, then utilizing the common Ethernet access, typically at 1 Mbytes/sec, the transfer time drops to 1 week. If the astronomer is logged on to the machine, which contains the data, all of it on hard disk, then using the normal SCSI bandwidth it still takes 1 day to scan through the data. We can conclude, that even faster hardware cannot support hundreds of 'brute force' queries per day, if no intelligent solution is used. With Terabyte catalogs, customized data sets of a few percent are still in the 10 GB range, thus an intelligent, high level data management is needed.

Current efforts under way, which together will create a unified multi- wavelength view of the Universe, include 2MASS, SDSS, POSS-2, FIRST, COBE, MAP, GSC-II, ROSAT and GALEX, with wavelengths from X-rays through UV, optical, near-IR to microwave and radio. Just the catalogs listed here will provide all-sky (or almost all-sky information in 15 different bands. Their main characteristics are that they have been designed to be homogeneous, are well calibrated and have a good control over systematics. The catalogs will typically contain over 100 million objects/pixels each, and will be multiple Terabytes in size. The overlap between them will be substantial, but at the same time there will be a lot of non-detections between different archives. Once these catalogs are completed, their combination will allow unique queries.

Soon there will be an enormous pressure from the whole astronomical community to integrate these separate archives into a seamlessly interoperating entity. The public access of such amounts of data, the necessary integration of the geographically distributed data sets and their interoperability poses non-trivial problems, some of which are technical, some are political and some are financial. Since most of these archiving projects are just about to begin, it is not too late to coordinate their architectures, but action must be taken very shortly. New standards for the exchange of large amounts of data need to be developed, and one has to address how to deal with the distributed nature of the data. We also need to consider that the computing platforms on which data are stored are heterogeneous, and evolve rapidly. Political complications include the facts that different data sets were created under the umbrella of different federal agencies (NSF, NASA).

\section{Main Functions of the Virtual Observatory}

Today we understand, that this process of integrating ther archives is inevitable, and within the next few years we will be faced with concrete challenges. In this paper we address the next relevant issue, what are the functionalities that these archives need to provide, and what are the fundamental standards that we need to define before we can continue on developing the tools. 
First of all, the data in the archives must be maintained, curated. We envisage that data would reside with the respective groups, who know their own data best. This of course means that the data is scattered across the continent. The groups would maintain their own data, and provide the storage, the documentation and the metadata, describing the contents of the respective archives.

There are different types of users, whose support requires a widely diverse array of resources. Much of the general astronomy public will only use these archives on a causal lookup basis, which will represent a www interface, and a large number of rather simple queries. These can be relatively easily supported via a central web-site with a state of the art, but not too complex query engine. Intermediate users will want to use the archive in a more elaborate fashion. Much more difficult is to serve the power users, who would require multiple searches in Terabytes of data, and extract hundreds of Gigabytes for further processing. This task much resembles accessing supercomputer resources, and our straw-man idea is to handle it like such.

We expect that most of the queries will be of exploratory nature, no two queries will be exactly alike, at least for a while. Generally, scientists will try to explore the multi-color properties of the objects in the catalog, starting with small queries of limited scope, then gradually making their queries more complex on a hit-and-miss basis. Several typical types of activities need to be supported: manual browsing, where one would look at objects in the same general area of the sky, and manually/interactively explore their individual properties, the creation of sweeping searches with complex constraints, which extend to a major part of the sky, searches based upon angular separations between objects on the sky, cross-identifications with external catalogs, creating personal subsets, and creating new "official" data products.

The data are inherently multidimensional, each object is represented by several fluxes, position on the sky, size, redshift, etc. Searching for special categories of objects, like quasars, involves defining complex domains in this $\mathrm{N}$-dimensional space. Spatial relations will be investigated, like finding nearest neighbours, or other objects satisfying a given criterion within an angular distance. The output size of the objects satisfying a given query can be so large that intermediate files simply cannot be created. The only way to analyze such data sets is to send them directly into analysis tools, thus these will have to be linked to the archive itself.

The interconnection of the different sites could be accomplished via one of the testbed programs connected to the next generation of high-speed networks. The networking technology for the high speed connectivity is here, but currently there few real-world applications, which can generate a serious network load. The Virtual Observatory could become a prime example of such creative network usage.

The creation and maintainance of the integrated framework involves several components. First, one needs to understand the requirements. Then appropriate standards need to be defined, then the tools conforming to those standards, interfaces and protocols must be built. Creation of the tools will require original development, by implementing state of the art techniques from Computer Science. The results will be very relevant much beyond astronomy, the whole 
society is struggling with information retrieval. This development can only be done by a wide collaboration, that involves not only astronomers, but computer scientists and statisticians and even participants from the IT industry.

\section{Discussion of the Common Standards}

\subsection{Communication Protocol}

Whenever people meet, they must agree (either implicitly or not) on a protocol that they will use to share information (i.e. a language). This description also applies to the connection of disparate archives and services, where the most basic requirement is that they must be able to communicate amongst themselves. This requirement can be satisfied by defining a standardized communication protocol which the individual components agree to employ. Such a protocol goes beyond a query language (which is a subset), and must provide support for command functionality and performance monitoring, for both inter-archive as well as archive-service communications.

The command functionality encompasses the needs for sharing metadata and metaservices between archives and services, allow archives to communicate amongst themselves, and monitor data streams (analysis filtering). In any complex, distributed archival system, the ability to perform basic checkpoint operations on a query: stop, pause, restart, and abort, is essential. Equally important is the ability to provide feedback to the end-user (either a monitoring process or the actual astronomer) on the status of the query. An additional benefit of a well-defined communication protocol is that pre-existing or legacy archival services can be retrofitted (by mapping the new standard onto existing services) in order to participate in collaborative querying.

In a collaborative query model, a multi-wavelength search (i.e. "Find all blue galaxies around dusty quasars") will be distributed to multiple archival centers. As a result certain optimizations can be performed depending on the status of the archival connections (network weather) in order to balance the resulting server load. Eventually, a learning mechanism can be applied to analyze queries, and using the accumulated knowledge gained from past observations (i.e. artificial intelligence), queries can be rearranged in order to provide further performance enhancements.

A promising candidate for implementing the communication protocol is the extensible markup language (XML). Different commands would be encoded using different XML tags whose definitions are standardized within a formal document type definition (DTD). The DTD would specify the appropriate action for a given command, such as how to combine multiple data streams and where the result should be sent.

\subsection{Hierarchical Metadata}

Continuing the previous analogy, the next step is to exchange relevant descriptive information. For archives, this primarily consists of their metadata (or data that describes the contents of the archive). For services (which can be contained within an archive or stand alone), it describes the specific nature of the work that the service can perform (such as cross-identification or image registration) 
and the expected format of the input and output data. Depending on the need of the consumer, different amounts (or levels) of detailed information might be required. For example, when announcing its availability to other archival centers, an archive might provide very general information concerning the specific wavelengths of the data that it contains and the areal extants that it covers. A specific user, however, might desire more detailed information, such as the type of detectors used during the observations, or the filter transmission efficiencies.

The nature and format of astronomical metadata clearly needs to be standardized. A hierarchical format will enable users to "drill down" until they reach the level of detail they require to perform their analysis. We feel that this another area that XML might prove beneficial. Not only is the hierarchical requirement satisfied, but it is easily generated, and can be parsed by machines and read by humans with equal ease. By adopting a standardized DTD, metadata can be easily archived and accessed by any conforming application.

\subsection{High Performance Streaming Interface}

In the traditional exploration model where small numbers of astronomical sources were analyzed, plain ASCII text was generally sufficient for passing data between sites. With the advent of large areal, digital surveys, however, the overhead of this approach severely taxes the performance of a system. What is required is a flexible system, in order to pass different types of data (i.e. tabular, spectral, or imaging data) that operates in a streaming fashion (similar to MPI), so that analysis of the data does not need to wait for the entire dataset before proceeding. As an example, we might need to register images, or cross-identify sources from different surveys.

A standardized binary data interchange format (the FITS-Flexible Image Transport Standard) currently exists within the astronomical community. The true efficacy of this format as a high performance streaming interface is unclear. Another option is XML wrapped binary transfers (which could employ a FITS approach).

\subsection{Efficient Object Interchange Format}

The primary purpose in federating multi-wavelength astronomical surveys is to combine all available information for a set of astronomical sources. In order to analyze the data streaming from multiple archives, a conceptual "object" identity is required for each unique source (i.e. the information that this source in archive $\mathrm{A}$ is that source in archive B). As a result, a standard format for efficiently interchange of astronomical "objects" is necessitated.

Traditionally this is accomplished by flattening the appropriate data into a file (e.g., the FITS Standard) and inflating the data at the other end of the stream. However, this is not necessarily the best method, and alternative techniques, such as serialization (using either Java or $\mathrm{C}++$ ) or an XML approach may prove more fruitful. The adoption of a standard interchange format would shorten the development cycle for user interface and analysis tools while extending their applicability. 


\subsection{Source Cross-Identification}

The cross-identification of billions of sources in both a static and dynamic state over thousands of square degrees in a multi-wavelength domain (Radio to XRay) is clearly an important and complex issue. This "join" operation is accomplished by cross-identifying sources in one archive with sources in multiple other archives. The process is, of course, much more complicated than it initially appears, due both to the nature of the data collection as well as the nature of astronomical objects. Observational data is always limited by the available technology, which varies greatly in sensitivity and resolution as a function of wavelength (e.g., optical instrumentation is generally superioir to infrared instrumentation). The calibration of the data (either spectral, temporal, or spatial) can also vary greatly. As a result, it is often very difficult to unambiguously match sources between different wavelength surveys; and, therefore, an association often has an assigned probability and possibly more attributes which solely describe the cross-identification object.

This problem becomes even more complicated when the large area of many surveys, and the subsequent billions of objects, are utilized. Furthermore, the facts that surveys have different limiting flux sensitivities and that astronomical objects generally do not have flat spectral shapes, implies that objects are often detected in one wavelength region, might be much fainter, brighter, or even not detected at all in another wavelength region (i.e. sources can look quite different depending on the wavelength region used). To overcome this difficulty, a priori astronomical knowledge concerning the spectral shape of "known" sources can be incorporated in order to optimally determine source associations. A major concern with this type of approach, however, is to prevent the misidentification of novel sources. The limited spatial resolution of some surveys, sometimes results in large positional error boxes, which can often result in one-to-many or even many-to-many associations. The task is also made more complicated by the areal extant of the cross-identification (i.e. full sky versus small areas). We also need to be able to encapsulate the domain knowledge that was used to determine the cross-identifications and their associated probability.

We see the need for standardization on two items: cross-identification objects, and cross-identification generators. By defining a format for encapsulating the astronomical knowledge that sources should be associated, we can persist this newly generated relationship data and begin to develop the often dreamed virtual observatories, where users see a seamless multi-wavelength view of the universe. On the other hand, some users will want to apply their own ideas to the problem of cross-identification (perhaps incorporating new domain knowledge). This will require a standardized interface for the new association generators so that they might easily be incorporated into existing frameworks (i.e. a plugand-play approach).

\subsection{Sky Partitioning and Navigation}

The largest interoperability issue has to do with standards and organization. As the volume of catalogued data in these archives will approach a Terabyte, to ensure the integrity of the data it is prudent to subdivide it into considerably smaller partitions, storing the subdivisions as separate files. This partitioning should utilize spatial information to a certain extent, something which has been 
done by several existing surveys, either by simulating the POSS plates, e.g. ROSAT, or utilizing their own partitons. Largely, these subdivisions are hidden from the user, thus one could argue that it does not matter how a catalogue is organized internally, as long as it provides a common functionality. On the other hand this requires a lot of parallel effort, and there are other bottlenecks on the horizon, where a common subdivision scheme may offer a lot of benefits. The first point of common interest is the definition of common areas over the sky, which can be universally used by astronomical databases. The need for such a system is indicated by the widespread use of the ancient constallations - the first spatial index of the celestial sphere. The existence of such an index, in a more computer friendly form would substantially speed up cross referencing between future catalogs, and would also enable a more consistent low-level organization between different catalogs. The entire sphere would be uniquely covered by these common regions. The boundaries of each area and the area's nomenclature could be fixed for the foreseeable future. A scheme for such subdivisions has been advocated earlier (Bartlett 1994). Here we would like to adopt this idea, and carry it one step further.

There is an obvious complication with this (or, for that matter, with any) approach: the density of objects is not uniform over the sky. Furthermore, the densities of different types of objects (and from different types of surveys) follow different distributions. For example, optical catalogs, primarily focusing on galaxies, like the SDSS, will target areas away from the plane of the Galaxy. Infrared surveys, like 2MASS, which cover the whole sky will have most of their objects close to the Galactic plane. Generally, an efficient partitioning of an archive will require an approximately balanced distribution of the number of objects per partition, a strategy already used by GSC-I. Having a common partitioning scheme, that provides a balanced partitioning for all catalogs involved simultaneously, may seem to be impossible, but as we will show below, there is an elegant solution, a 'shoe that fits all' — by subdividing the sky in a hierarchical fashion!

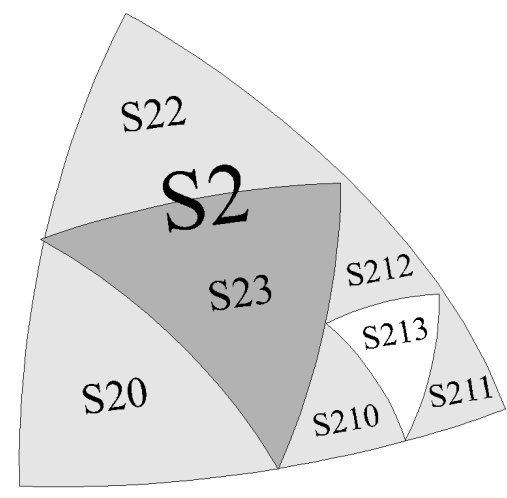

Fig. 1. An illustration of how a spherical triangle S2 is subdivided into four smaller ones. We also indicate how one of these, S21, is subdivided further into S210...S213. Note, how the name keeps track of the parents of a given region. 
Our approach to dealing with this complication is the following: instead of taking a given subdivision, we specify an increasingly finer hierarchy of subdivisions, where each level is fully contained within the previous one. There would be a 'base set' of selected areas. Each selected area can, if necessary, be divided into a number (proposed to be 4) of approximately equal sub-areas. Each subarea can be divided further into an additional 4 sub-areas, ad infinitum. Such hierarchical subdivisions can be very efficiently represented on the computer in the form of quad-trees (Samet 1990, Fekete 1990).

Each base selected area would be assigned a name, perhaps something simple like S2 ( $\mathrm{S}$ for South, 2 to identify one of the four). If an archive has too many objects contained within S2 to handle comfortably, it would divide S2 into sub-areas: S20, S21, S22, and S23, for example. If S21 contained too many objects, it could be further subdivided into four more equal sub-areas designated S210, S211, S212 and S213, etc. This number, on how deep a particular area would be subdivided in a given catalog, will be a function of the storage size of the entry per object in the given database.

This way, any of these selected areas are uniquely specified by their name, even within the hierarchy - the name will tell which area is the parent, also how deep we are in the hierarchy. In such a way areas in different catalogs map either directly onto one another, or one is fully contained by another.

For Cosmic Microwave Background experiments various similar pixelization schemes have been proposed (Saff and Kuijlaars 1997, Greisen etal 1993, Tegmark 1996, Gorski 1997, Crittenden and Turok 1998). Mostly, these schemes are not hierarchical, rather they create a grid of a given resolution, which can then be optimized for various properties, like having a lateral symmetry for fast spherical harmonic computations, or equal area, usually at the expense of another property, like similarity of shape.

One can create a hierarchical pixelization scheme starting from an arbitrary Platonian solid, like tetrahedron, cube, dodecahedron (Fekete 1990), or icosahedron (Tegmark 1996). Each of these have emerged in the literature. The faces of these solids are regular polygons, and their projections on the unit sphere is hierarchically subdivided further, using great circles. Such subdivision schemes are well known in computer graphics (Ahn 1995).

Contrary to common wisdom, we propose to store the coordinates in a Cartesian form, i.e. as a triplet of $x, y, z$ values per object. The $x, y, z$ numbers represent the position of objects on the unit sphere. While at first this may seem to increase the required storage (three numbers per object vs two angles), it makes querying the database for objects within certain areas of the celestial sphere, or involving different coordinate systems considerably more efficient. Again, this is by no means new, this was used highly successfully by GSC-I.

The coordinates in the different celestial coordinate systems (Equatorial, Galactic, Supergalactic, etc) can be constructed from the Cartesian primitive, on the fly. Conversions are performed by standard functions which convert between the angular and cartesian coordinate objects. This approach allows the conversion to be specified at run time, allowing recalibrations to be seamlessly integrated.

The most common types of queries involving celestial coordinates are of the four fundamental types listed below. As we will see, having our coordinates 
stored as three-dimensian vectors will make the evaluation of all these queries considerably easier. Consider the fact, that all objects above a given latitude in a certain coordinate system can also be looked at as the objects on one side of the plane in $3 \mathrm{~d}$ space, which corresponds to the small circle of the latitude. The four fundamental query types are the following:

- all objects within a certain spherical distance of a given point. These can be recast into intersections on the unit sphere with a plane, with the plane normal pointing at the center.

- objects above a given latitude in any spherical coordinate system are above a three dimensional plane, intersecting the sphere at that latitude circle.

- any great circle, thus any longitude is represented by the intersection of the sphere with a plane going through the origin.

- Boolean combinations (AND, OR, NOT) of the above

All these primitive queries are so-called half-space queries, where each of the constraints excludes half of the three dimensional space (but not half the sky!). Evaluating these queries is very inexpensive in terms of CPU cycles, since they all involve a computation of a linear combination rather than a spherical distance for example. Also, even though the query may contain constraints specified in several different coordinate systems, these coordinates need not be stored or even evaluated for each object, only linear combinations of the Cartesian are evaluated for every constraint. In this way, every spherical coordinate system is handled in the same fashion. Besides, these linear combinations with their Boolean operations can trivially be represented in query languages like SQL or OQL.
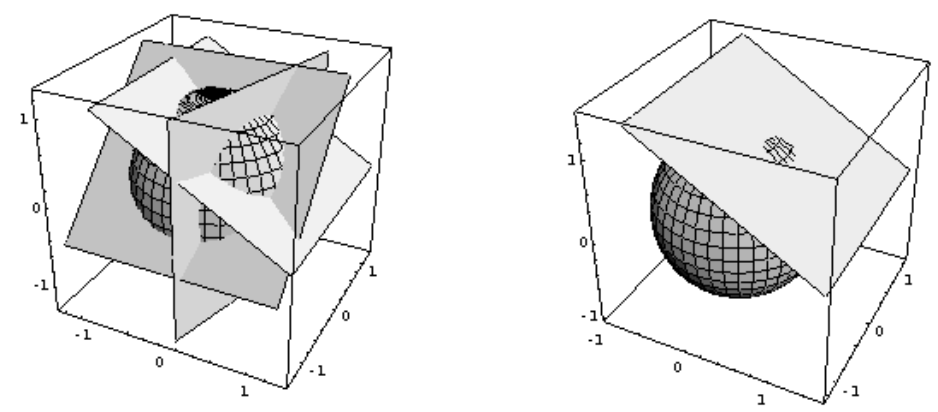

Fig. 2. Schematic representation of how the 3-dimensional Cartesian representation of coordinates make queries like objects within a certain spherical distance to a given point, or combination of constraints in multiple coordinate systems become particularly simple, since they correspond to testing linear combinations of the Cartesian coordinates instead of complicated trigonometric expressions. 


\subsection{Uniform Handling of Detections and Upper Limits}

The advantage of using a logarithmic scale to measure astronomical fluxes is obvious, the magnitude scale is able to span a huge dynamic range. When relative colors are needed, they can be computed by differencing magnitudes measured in different bandpasses. These advantages are quite clear for bright objects, where noise is not an issue. On the other hand, as fluxes become comparable to sky- and instrumental noise, the magnitudes become more and more scattered due to the singularity in the magnitude scale at zero flux. The cases, where due to a downward sky fluctuation we measure negative flux for an object cannot be represented with magnitudes. People have generally handled these cases by specifying detection flags, and encoding the negative flux somehow. These problems become even more pronounced as we work in multicolor space. An object can be well detected and measured in several of the bands, and still fail to provide a measurable flux in others. This information of non-detection is meaningful, beyond just a detection flag, in flux space the object would be represented as a multi-variate Gaussian probability centered on the flux values, not necessary all positive. In magnitude space, the error ellipsoid of such an object would have an infinite extent in some of the directions, making meaningful multicolor search criteria in a database extremely complex, if not impossible. All the non-detections in one or more bands have to be isolated, and treated separately. One can conclude, that most problems arise from the singular behaviour of the logarithm function. We will discuss here a suggestion by Jim Gunn and Robert Lupton, to keep all the advantages of magnitudes, without their disadvantage. We expect that this will be the way how fluxes will be represented in the SDSS Science Archive.

We propose to use the inverse hyperbolic sine function instead of the logarithm, in defining a new magnitude scale. This function behaves as the logarithm for large values of its arguments, while staying linear close to zero.

$$
\sinh ^{-1}(x)=\ln \left[x+\sqrt{x^{2}+1}\right] \rightarrow \begin{cases}\ln 2 x, & \text { if } x \gg 1 \\ x, & \text { if }|x|<1\end{cases}
$$

Let us consider the dimensionless, normalized flux $x$, with a correct zero-point, defined as $x=f / f_{0}$. The usual apparent magnitude $m$ can be written as

$$
m=-2.5 \log _{10} x=-2.5 / \ln 10 \ln x=-a \ln x .
$$

We can also define the new magnitude $\mu$ as

$$
\mu(x)=-a\left[\sinh ^{-1}\left(\frac{x}{2 b}\right)+\ln b\right]
$$

Here $a$ and $b$ are constants, $a=2.5 / \ln 10=1.08574$, and $b$ is an arbitrary 'softening', set to the optimum value of $b=\sqrt{a} \sigma=1.042 \sigma$, where $\sigma$ is the typical error in a object's flux. The value of $b$ determines at which flux level will

the linear behaviour set in. Consider the asymptotic behaviour of $\mu$, for both high and low $x$ :

$$
\lim _{x \rightarrow \infty} \mu(x)=-a \ln x=m \quad \lim _{x \rightarrow 0} \mu(x)=-a\left[\frac{x}{2 b}+\ln b\right] .
$$


Thus for $x \rightarrow \infty, \mu$ approaches correctly $m$, for any choice of $b$. Now consider the low $x$ asymptotic behaviour, and see that it is indeed linear in $x$, but this behaviour does depend on $b$.

We have to take into account, that all the flux measurements will have an error. Differences which are much smaller than the flux error do not matter much. Assume that the normalized flux $x$ has a Gaussian error $\sigma^{2}=\left\langle x^{2}\right\rangle-\langle x\rangle^{2}$. Let us compute the variances for the two magnitudes and their difference. The arrows indicate the asymptotic behaviour for $x \rightarrow 0$.

$$
\begin{array}{rccc}
\left\langle\Delta m^{2}\right\rangle= & \frac{a^{2} \sigma^{2}}{x^{2}} \rightarrow & \frac{a^{2} \sigma^{2}}{x^{2}} \\
\left\langle\Delta \mu^{2}\right\rangle= & \frac{a^{2} \sigma^{2}}{4 b^{2}+x^{2}} \rightarrow & \frac{a^{2} \sigma^{2}}{4 b^{2}} \\
m-\mu= & a \ln \left[\frac{1+\sqrt{1+4 b^{2} / x^{2}}}{2}\right] \rightarrow & -a \ln \left(\frac{x}{b}\right)
\end{array}
$$

In the discussion above, we considered the idealized case, that all objects have the same Gaussian error, mostly dominated by sky noise. This of course assumes, that all the apertures in the survey are the same. Also, the actual noise may vary with the position on the sky, like the vicinity of a bright object, etc. Therefore, we need to distinguish between the nominal and actual scatter in a given object's flux. The nominal dispersion is the one averaged over all objects in the survey, dominated by the faintest objects at the edge of detection. This quantity is an average one. The actual dispersion is arising indeed from local noise, varying aperture sizes, etc. The two will only differ for relatively big and bright objects, or for small objects sitting on the wings of bright stars.

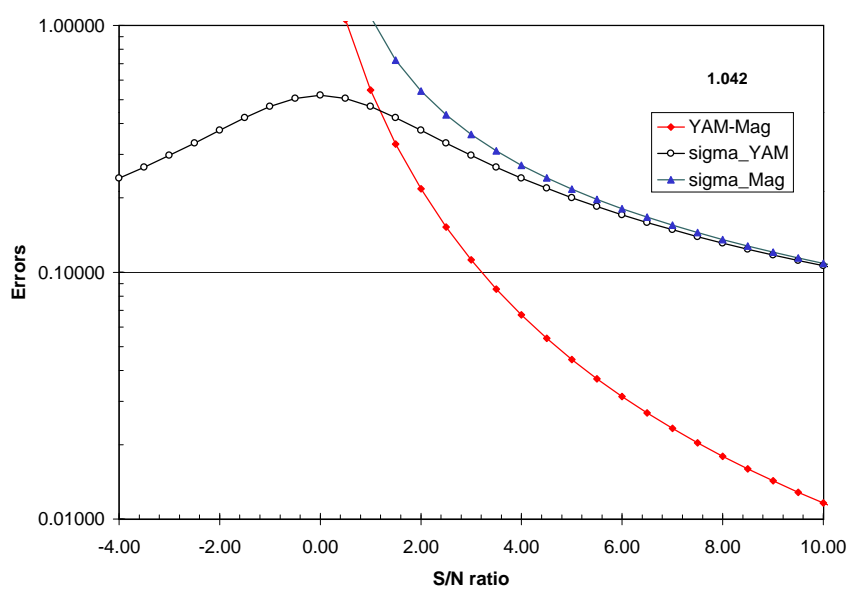

Fig. 3. We show the variances and the difference as a function of the signal-tonoise $x / \sigma$. The open circles represent $\Delta \mu$, the filled triangles represent $\Delta m$ and the filled diamonds correspond to $m-\mu$. The figure corresponds to the optimal choice of $b=1.042$, as discussed below. Note, how small the deviation $m-\mu$ 
is compared to the magnitude error. For a $\mathrm{S} / \mathrm{N}$ of 3 , the difference is less than 0.1 , while $\Delta m=0.3$.

We suggest, that one global choice of $b$ be adopted for each of the filters for a given survey, which correspond to the mean nominal dispersion of the faintest objects in each band. Then the actual fluxes can be recovered with a global inverse transformation as needed. Photometric zero points can still be handled as usual, since they occur at such a high flux level, that the difference between the two magnitudes is a fraction of a percent. Multicolor searches would then deal with objects, which have well-localized error ellipsoids. The error ellipsoids can follow the actual dispersion, which can then exceed the nominal maximum value of 0.52 . If an object is not detected in a given band, its error ellipsoid (of about 0.52 magnitudes) would be centered on the value corresponding to zero flux, but the object would be still very well localized.

\subsection{Advanced Query Language}

In typical astronomical queries the users fill out a www form, with minimal constraints, like a point on the sky and a search radius, or a name of a well known object, or a right ascension and declination range, combined with a magnitude range. For more complex science, in particular for finding interesting objects with unusual colors, one needs much more complex constraints. In the process of classifying different types of objects, typically rather complex decision surfaces are needed, most often in a higher dimensional space. Like in order to find quasars, we need objects which are point-like, and have unusual colors, which can be both very blue or very red, depending on the redshift of the quasars. Such decision surfaces are very complicated. How can these be represented? We would like to promote the idea, that by allowing linear combinations of magnitudes, one can create a multidimensional half-space query. Their Boolean combinations, similar to our ideas for the sky navigation, are multidimensional polyhedra. These can be expressed in the more advanced formal query languages, like SQL or OQL.

There are of course queries, which will be required for advanced astronomical searches, which involve spatial relations, proximities between objects. These cannot be expressed by traditional SQL expressions, we need new extensions to the language. These extensions should contain a spherical distance operator between two objects, and a distance based on a Eucledian metric in magnitude space. Such an operator needs to have not only a proximity radius in magnitude space, but a description of the subspace the distance should be calculated in. Such a query could be: Find me all galaxies, which have another one within 10 seconds of arc, with colors within 0.2 magnitudes in $r$ and $b$. The ability of embedded SELECT statements is a necessary condition to be able to describe the complex crieteria. Thus, we envisage that the astronomy community will create a small number of common extensions to SQL, describing now functions with a spatial content, and most of the archives will adopt these.

Searching distributed archives with a single query statement is much more complex. One can possibly accommodate this by

- either using such simple queries, that only relate to attributes common to all archives 
- include specific scope statements in the FROM clauses relevant for each of the archives, by keeping track of the individual data models

- by creating a federated data model, that is the union of all the data models in the Virtual Observatory, and preprocess the query using this knowledge

- via an intelligent federated layer, that given a query, interrogates the archives about their contents and distributes the query accordingly

This is an area that will require a lot of further research.

There will also be queries which will only return aggregate quantitites, like tyhe number of objects with a given property, or the average of an attribute, given a set of constraints. Such queries may be executed very efficiently if we have a condensed representation of the data. Condensed representations enable one to retrieve such aggregate information without performing the detailed query, and are an active area of modern computer science (Moore 1998).

\subsection{Multiepoch observations}

Many astronomical objects are variable, and observations made at different times may thus give different results about their brightness. Other objects, like asteroids are mnoving rather rapidly on the sky. It is important to keep track of the information when the respective observations were made. It should be easy to extract all observations of a given object, in all archives, ordered in sequence. One can then develop various hypotheses about the nature of their variability (like what is the significance that this is an RR Lyrae variable). There may be several hypotheses equally likely. These qould be stored in a database. As further observations become available, one can reexamine and update these hypotheses, refine their parameters.

A similar approach could be done with asteroids - from their first few observations one can already calculate a crude trajectory. As at another time a new asteroid is observed, first one should check, whether it is consistent with any of the previously detected asteroids, by brightness and trajectory, and if so, one can refine those models. If no match is found, a new hypothesis is calculated and inserted into the database.

On the time series one can create increasingly refined models for certain classes of objects, like variable stars. One can eventually extract light curves, possibly in multicolor, or tag single transient events like supernovae. This is an area of astronomy which is in rapid development, but most of these queries have been restricted to datasets covering a small part of the sky.

\section{Summary}

In this paper we listed, without the sake of completeness some of the important problems facing the next generation archives in astronomy. The future lies in their seamless integration, but there is lot to be done, the Virtual Observatory may be just as complex if not more so than a traditional one. However, astronomy will be very different ten years from now, much more research will be done via the integrated archives, and data mining will be an integral part of every astronomer's daily routine, just as using a web browser and email is today. 
Acknowledgments. We wish to thank George Djorgovski, John Good, Gretchen Greene, Barry Lasker, Tom Prince, Doug Reynolds, Gyula Szokoly, Ani Thakar, and Roy Williams, for stimulating discussions.

\section{References}

Szalay, A.S., Brunner, R.J., Connolly, A., Pier, J., York, D., Lonsdale, C., Good, J., Lasker, B., Greene, G., and McLean, B., 1998, in preparation.

Szalay,A.S. and Brunner,R.J., in New Horizons from Multi-Wavelength Sky Surveys, IAU Symposium 179, eds. B.McLean and D.Golombek, p.455 (1997)

Bartlett,P. Proc. ADASS, 1994.

Samet,H., Applications of Spatial Data Structures, Addison Wesley (1990)

Fekete,G. Ph.Thesis, The University of Maryland (1990)

Saff,E.B. and Kuijlaars,A.B.J., The Mathematical Inelligencer, 19, 1, p5 (1997)

Greisen,E.W. and Calabretta,M. Bull.Am.Astron.Soc. 182, 09.01 (1993)

Tegmark,M. Ap. J. Lett. 470, L81 (1996)

Gorski,K. private communication (1997)

Crittenden,R.G. and Turok,N.G. astro-ph/9806374 (1998)

Ahn,J. Graphics Gems V., Ed: A.W. Paeth, AP Professional, p.179 (1995)

Moore, A.W. and Lee, M.S., Cached Sufficient Statistics for Efficient Machine Learning with Large Datasets, Journal of Artificial Intelligence 8, (1998)

Lasker,B.M. B.J.McLean, H.Jenkner, M.G.Lattanzi and A.Spagna, Proceedings of the Workshop 'Future Possibilites for Astrometry in Space', p137. ESA Sp-379 (1995) 